\title{
A Novel Cohesinopathy Causing Chronic Intestinal Pseudo Obstruction in 2 Siblings and Literature Review
}

\section{Introduction}

Chronic intestinal pseudo obstruction (CIPO) is a rare intriguing disorder affecting the gastrointestinal (GI) motility leading to significant morbidity and mortality. As the name indicates, affected children experience symptoms of intestinal obstruction in the absence of any mechanical cause. ${ }^{1}$ Various genes like SOX10, FLNA, L1CAM, TYMP, and POLG are implicated in the pathogenesis of familial forms of CIPO ${ }^{2}$ Here we describe 2 siblings of the same family affected with CIPO and identified to harbor mutation in the Shugoshin like-1 (SGOL1) gene on genetic analysis.

\section{Case}

The index patient is a 12-year-old boy, born out of a nonconsanguineous marriage, who presented with constipation, intermittent episodes of pain, and distension of the abdomen for a year associated with poor weight gain. X-rays and CT scans of the abdomen done on various occasions showed multiple air fluid levels, dilation of small bowel loops, normal sized ureters (Figure A-C), and was managed as subacute intestinal obstruction. The hemogram, liver, kidney, and thyroid function tests were normal. Esophagogastroduodenoscopy revealed a dilated body of stomach
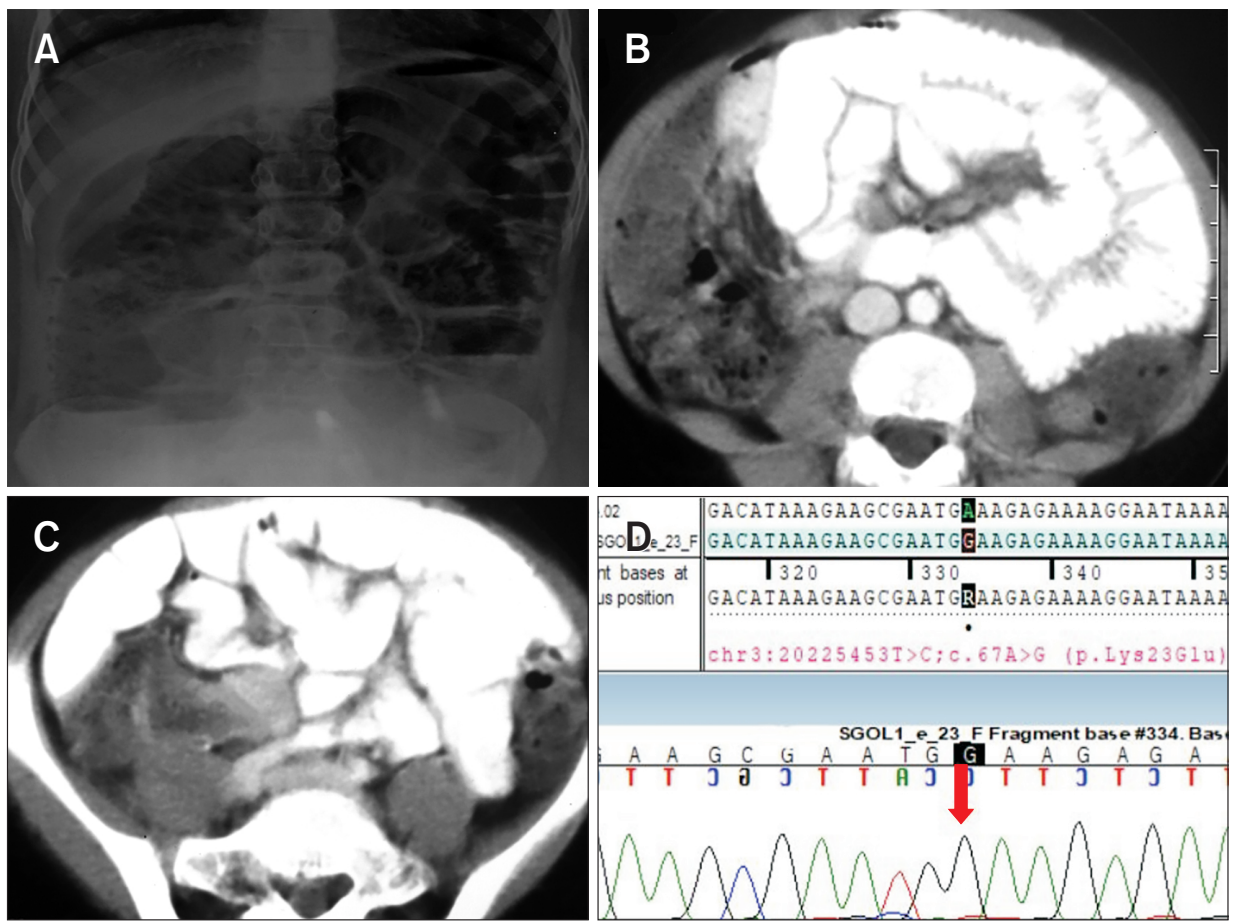

s position

GACATAAAGAAGCGATGRAAGAGAAAGGAATAAAA chr3:20225453I>C;C.67A >G (p. Lys23G1u



Figure. (A) X-ray of the abdomen showing dilated bowel loops with air fluid levels. Note can be made of fecal loading of the colon. (B, C) CT scan of the abdomen showing dilated small bowel loops. No obvious mechanical cause of obstruction could be found. (D) Sequence chromatogram showing variation in exon 2 (c.67A>G; red arrow) of the Shugoshin like-1 (SGOL) gene in the index patient. 
with bile reflux, dilated duodenum, and ileocolonoscopy was normal. The biopsy samples from the esophagus, stomach, ileum, and colon were normal on histopathology. High-resolution esophageal manometry showed weak peristalsis with small peristaltic breaks; anorectal manometry was normal. A whole bowel transit study revealed normal gastric emptying with delayed colonic transit time. There was only a partial response with medical therapy (polyethylene glycol and itopride). Age-appropriate protein-calorie diet with supplemented vitamins and micronutrients was advised. Similar complaints and investigative findings were noted in his elder sibling, a 16-year-old girl who presented with constipation, pain abdomen for 3 years. Neither of the siblings reported palpitation, dizziness or syncope, and the cardiac evaluation (12 lead electrocardiogram and echocardiography) of both was normal. Due to familial occurrence, a possibility of primary CIPO was suspected and next generation sequencing performed in the index patient revealed a homozygous missense variation in exon 2 of SGOL1 gene (chr3; c67A>G; p.Lys23Glu; Figure D). It was later validated by Sanger sequencing and the same mutation was also identified in the sibling.

\section{Discussion}

Small bowel and colon are commonly involved segments in children with CIPO, though it can involve any part of the GI tract. Due to its rarity, lack of knowledge regarding the disease entity, and absence of well-defined diagnostic criteria, the diagnosis of CIPO is often delayed. ${ }^{3}$ Moreover, lack of definitive pharmacological treatment results in significant morbidity impairing the quality of life, leading to intestinal failure and death. ${ }^{4,5} \mathrm{~A}$ proportion of primary CIPO in children is familial. SGOL1 gene encodes for a part of cohesin complex which is involved in cell division and is expressed in the intestinal wall; both smooth muscle and enteric nervous system. On extensive literature search, we identified only 2 studies describing CIPO due to SGOL1 mutations. In a single study of 17 patients, the authors describe a syndrome termed chronic atrial and intestinal dysrhythmia due to homozygous mutations in SGOL1 that cause failure of cohesin protection, promoting cell senescence leading to hypoplastic, mislocalized ganglia and Cajal cells in the smooth muscle. ${ }^{6}$ Poinsot et al, ${ }^{7}$ reported CIPO due to SGOL1 mutations presenting at an older age with predominant GI manifestations and absence of cardiac involvement as seen in our case. ${ }^{7}$ Small bowel motility testing could not be performed in our patients. There was no history of consanguinity in the parents of these children but they belong to the North Indian "Baniya or Aggarwal Community," who tend to be highly endogamous and founder variations in culpable genes of some genetic disorders are described in this community. ${ }^{8}$ Patients with this disorder gradually develop intestinal failure requiring total parenteral nutrition. At the time of writing this report, both siblings were on medical treatment and are under periodic follow-up for assessing the need of total parenteral nutrition as well as for any evidence of cardiac dysfunction. To the best of our knowledge, this is the first report from Asia of this gene mutation leading to CIPO.

Vybhav Venkatesh, ${ }^{1}$ Aradhana Aneja, ${ }^{1}$ Keerthivasan Seetharaman, ${ }^{1}$ Neha Anushree, ${ }^{1}$ Surinder S Rana, ${ }^{2}$ and Sadhna B Lal ${ }^{1 *}$ ${ }^{1}$ Division of Pediatric Gastroenterology, Hepatology, and Nutrition, ${ }^{2}$ Department of Gastroenterology, Post Graduate Institute of Medical Education and Research, Chandigarh, India

1. Rudolph CD, Hyman PE, Altschuler SM, et al. Diagnosis and treatment of chronic intestinal pseudo-obstruction in children: report of consensus workshop. J Pediatr Gastroenterol Nutr 1997;24:102-112.

2. Di Nardo G, Di Lorenzo C, Lauro A, et al. Chronic intestinal pseudoobstruction in children and adults: diagnosis and therapeutic options. Neurogastroenterol Motil 2017;29:e12945.

3. Thapar N, Saliakellis E, Benninga MA, et al. Paediatric intestinal pseudo-obstruction: evidence and consensus-based recommendations from an ESPGHAN-led expert group. J Pediatr Gastroenterol Nutr 2018;66:991-1019.

4. Faure $\mathrm{C}$, Goulet $\mathrm{O}$, Ategbo $\mathrm{S}$, et al. Chronic intestinal pseudoobstruction syndrome clinical analysis, outcome, and prognosis in 105 children. Dig Dis Sci 1999;44:953-959.

5. Mousa H, Hyman PE, Cocjin J, Flores AF, Di Lorenzo C. Longterm outcome of congenital intestinal pseudoobstruction. Dig Dis Sci 2002;47:2298-2305.

6. Chetaille P, Preuss C, Burkhard S, et al. Mutations in SGOL1 cause a novel cohesinopathy affecting heart and gut rhythm. Nat Genet 2014;46:1245-1249.

7. Poinsot P, Castilloux J, Andelfinger G, Faure C. A170 A new pediatric intestinal pseudo-obstruction syndrome by sgol1 mutation: a late-onset but severe digestive phenotype. J Can Assoc Gastroenterol 2019;15;2(suppl 2):336-336.

8. Angural A, Spolia A, Mahajan A, et al. Review: understanding rare genetic diseases in low resource regions like Jammu and Kashmir - India. Front Genet 2020;11:415.

Acknowledgements: Written informed consent has been obtained from the parents of the patients reported in this article.

Financial support: None.

Conflicts of interest: None.

Author contributions: Vybhav Venkatesh, Aradhana Aneja, Keerthivasan Seetharaman, Neha Anushree: collection of patient data, clinical management of the patients and drafting the manuscript; Surinder S Rana and Sadhna B Lal: guidance on management of the patients, and reviewing and editing the final manuscript. 